\title{
A prospective, randomized, crossover pilot study of inhaled nitric oxide versus inhaled prostacyclin in heart transplant and lung transplant recipients
}

\author{
Tanveer A. Khan, MD, ${ }^{\mathrm{a}, *}$ Gabriel Schnickel, MD, ${ }^{\mathrm{a}, *}$ David Ross, MD, ${ }^{\mathrm{b}}$ Sam Bastani, DO, \\ Hillel Laks, MD, ${ }^{\mathrm{a}}$ Fardad Esmailian, MD, ${ }^{\mathrm{a}}$ Daniel Marelli, MD, ${ }^{\mathrm{a}}$ Ramin Beygui, MD, ${ }^{\mathrm{a}}$ \\ Richard Shemin, MD, ${ }^{\mathrm{a}}$ Larry Watson, BS, ${ }^{\mathrm{a}}$ Irina Vartapetian, BS, ${ }^{\mathrm{a}}$ and Abbas Ardehali, $\mathrm{MD}^{\mathrm{a}}$
}

\begin{abstract}
Objective: Inhaled nitric oxide has been shown to reduce pulmonary vascular resistance in patients undergoing cardiothoracic surgery, but it is limited by toxicity, the need for special monitoring, and cost. Inhaled prostacyclin also decreases pulmonary artery pressure, is relatively free of toxicity, requires no specific monitoring, and is less expensive. The objective of this study was to compare nitric oxide and prostacyclin in the treatment of pulmonary hypertension, refractory hypoxemia, and right ventricular dysfunction in thoracic transplant recipients in a prospective, randomized, crossover pilot trial.
\end{abstract}

Methods: Heart transplant and lung transplant recipients were randomized to nitric oxide or prostacyclin as initial treatment, followed by a crossover to the other agent after 6 hours. Pulmonary vasodilators were initiated in the operating room for pulmonary hypertension, refractory hypoxemia, or right ventricular dysfunction. Nitric oxide was administered at $20 \mathrm{ppm}$, and prostacyclin was administered at $20,000 \mathrm{ng} / \mathrm{mL}$. Hemodynamic and oxygenation parameters were recorded before and after initiation of pulmonary vasodilator therapy. At 6 hours, the hemodynamic and oxygenation parameters were recorded again, just before discontinuing the initial agent. Crossover baseline parameters were measured 30 minutes after the initial agent had been stopped. The crossover agent was then started, and the hemodynamic and oxygenation parameters were measured again 30 minutes later.

Results: Heart transplant and lung transplant recipients $(n=25)$ were randomized by initial treatment (nitric oxide, $n=14$; prostacyclin, $n=11$ ). Nitric oxide and prostacyclin both reduced pulmonary artery pressure and central venous pressure, and improved cardiac index and mixed venous oxygen saturation on initiation of therapy. More importantly, at the 6-hour crossover trial, there were no significant differences between nitric oxide and prostacyclin in the reduction of pulmonary artery pressures or central venous pressure, or in improvement in cardiac index or mixed venous oxygen saturation. Nitric oxide and prostacyclin did not affect the oxygenation index or systemic blood pressure. There were no complications associated with nitric oxide or prostacyclin.

Conclusion: In heart transplant and lung transplant recipients, nitric oxide and prostacyclin similarly reduce pulmonary artery pressures and central venous pressure, and improve cardiac index and mixed venous oxygen saturation. Inhaled prostacyclin may offer an alternative to nitric oxide in the treatment of pulmonary hypertension in thoracic transplantation. (J Thorac Cardiovasc Surg 2009;138:1417-24)

In heart transplantation, donor heart right ventricular (RV) dysfunction remains a major cause of early mortality. ${ }^{1,2}$ Elevated recipient pulmonary vascular resistance caused by chronic left-sided heart failure, adverse effects of cardiopulmonary bypass and protamine, and donor heart ischemia-reperfusion injury contribute to the donor heart RV dysfunction. Treatment strategies include optimization of hemodynamic, acid-base, and oxygenation status, and

From the Division of Cardiothoracic Surgery, Department of Surgery, ${ }^{\mathrm{a}}$ and Division of Pulmonary and Critical Care Medicine, Department of Medicine, ${ }^{b}$ UCLA School of Medicine, Los Angeles, Calif.

* T.A.K. and G.S. contributed equally to this work

Received for publication June 25, 2008; revisions received Feb 26, 2009; accepted for publication April 6, 2009.

Address for reprints: Abbas Ardehali, MD, Division of Cardiothoracic Surgery, UCLA School of Medicine, 62-232 CHS, Los Angeles, CA 90095-1741 (E-mail: aardehali@mednet.ucla.edu).

0022-5223/\$36.00

Copyright (c) 2009 by The American Association for Thoracic Surgery

doi:10.1016/j.jtcvs.2009.04.063 selective pulmonary vasodilator therapy. Similarly, primary graft dysfunction (PGD) remains a major obstacle in lung transplantation; it is a known risk factor for early and late death, and the development of bronchiolitis obliterans syndrome. ${ }^{3,4}$ PGD is characterized by pulmonary hypertension and hypoxemia. The standard treatment for PGD remains supportive therapy. Selective pulmonary vasodilator therapy is usually added to reduce pulmonary vascular resistance and improve oxygenation.

Despite its off-label use, inhaled nitric oxide (NO) has become a preferred agent in the management of pulmonary hypertension, RV dysfunction, and refractory hypoxemia in clinical heart and lung transplantation. ${ }^{5-9}$ Inhaled NO requires a special delivery device, is associated with toxicities that necessitate frequent monitoring, and is expensive. ${ }^{10} \mathrm{Be}-$ cause of these limitations of NO, several other agents have been examined as alternatives. Inhaled prostacyclin (PGI2) was first reported as a selective pulmonary vasodilator in 


$$
\begin{aligned}
& \text { Abbreviations and Acronyms } \\
& \mathrm{CI}=\text { confidence interval } \\
& \mathrm{CVP}=\text { central venous pressure } \\
& \mathrm{FIO}_{2}=\text { inspired oxygen fraction } \\
& \mathrm{NO}=\text { nitric oxide } \\
& \mathrm{PA}=\text { pulmonary artery } \\
& \mathrm{PaO}_{2}=\text { arterial oxygen tension } \\
& \mathrm{PGD}=\text { primary graft dysfunction } \\
& \mathrm{PGI}=\text { prostacyclin } \\
& \mathrm{RV}=\text { right ventricular } \\
& \mathrm{SVO}_{2}=\text { venous oxygen saturation }
\end{aligned}
$$

an experimental model in $1993 .{ }^{11}$ PGI2 is an arachidonic acid derivative with a half-life of 3 to 6 minutes. PGI2 binds to a prostanoid receptor, causing an increase in intracellular cyclic adenosine monophosphate and consequently vasodilation. Inhaled PGI2 has been shown to reduce pulmonary artery (PA) pressure and improve RV dysfunction and hypoxemia in a variety of pathologic states. ${ }^{12-16}$ To the best of our knowledge, there are no comparison studies of inhaled PGI2 versus inhaled NO in heart transplant and lung transplant recipients. The objective of this study was to compare the efficacy of NO with PGI2 in heart transplant and lung transplant recipients with pulmonary hypertension, RV dysfunction, or refractory hypoxemia. The primary end point of this pilot trial was the mean PA pressure. The secondary end points were central venous pressure (CVP), cardiac index, mixed venous oxygen saturation $\left(\mathrm{SvO}_{2}\right)$, mean systemic arterial pressure, and oxygenation index (arterial oxygen tension $\left[\mathrm{PaO}_{2}\right] /$ inspired oxygen fraction $\left[\mathrm{FiO}_{2}\right]$ ratio).

\section{MATERIALS AND METHODS}

This study was approved by the University of California Los Angeles Institutional Review Board. All adult patients undergoing heart and lung transplantation at the University of California Los Angeles were invited to enroll in this study preoperatively.

In heart transplant recipients, the decision to institute pulmonary vasodilator therapy was made by the attending surgeons at weaning of cardiopulmonary bypass. The criteria included a) pulmonary hypertension defined as mean PA pressure greater than $25 \mathrm{~mm} \mathrm{Hg}$ or 2) RV dysfunction defined as CVP greater than $12 \mathrm{~mm} \mathrm{Hg}$ with cardiac index less than $2.2 \mathrm{~L} / \mathrm{min} / \mathrm{m}^{2}$ and visual evidence of RV dysfunction. The standard inotropic regimen for weaning heart transplant recipients off cardiopulmonary bypass included epinephrine and milrinone. Pulmonary vasodilator therapy was instituted once all other hemodynamic and metabolic parameters were optimized. Patients were then randomized to NO or PGI2.

In single-lung transplant recipients, the decision to institute pulmonary vasodilator therapy was made at the time of clamping the PA intraoperatively or anytime thereafter. Development of pulmonary hypertension (defined as mean PA pressure $>25 \mathrm{~mm} \mathrm{Hg}$ ) or hypoxemia $\left(\mathrm{PaO}_{2} / \mathrm{FIO}_{2}\right.$ $<150$ ) prompted randomization to NO or PGI2. All double-lung transplant procedures were performed with the assistance of cardiopulmonary bypass. The decision to start pulmonary vasodilator therapy in double-lung transplant recipients was made at the time of weaning of cardiopulmonary

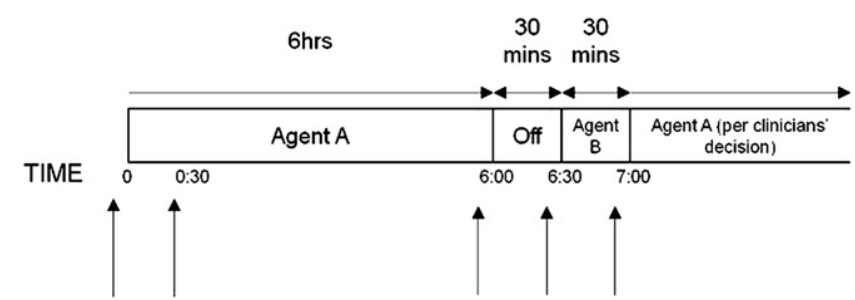

FIGURE 1. Time line for the experimental protocol and data collection. Patients were randomized to a pulmonary vasodilator, shown as Agent A, which was continued for 6 hours. Agent A was then stopped for 30 minutes for washout. Next, the crossover agent, shown as Agent B, was started and continued for 30 minutes. Therapy was then switched back to agent A, which was continued or weaned per the clinicians' decision. Time points (arrows) for collection of hemodynamic and oxygenation parameters.

bypass, in the setting of pulmonary hypertension or hypoxemia, as defined above.

\section{Experimental Protocol}

Once the decision was made to start pulmonary vasodilator therapy, the patient was randomized to either $\mathrm{NO}$ (at $20 \mathrm{ppm}$ ) or $\mathrm{PGI}_{2}$ (at 20,000 ng/ $\mathrm{mL}$ ). After 6 hours, the randomized agent was stopped for 30 minutes for washout, and the crossover agent was begun (Figure 1). The rationale for the 6-hour crossover trial was to allow sufficient time for the patient's hemodynamic and oxygenation parameters to stabilize. The crossover agent was administered for 30 minutes, followed by switching to the originally randomized agent. The duration of treatment and the weaning protocol was determined by the clinical team. Hemodynamic and oxygenation parameters were collected at time 0 (before starting the randomized agent), at 30 minutes (after starting the agent), at 6 hours (just before stopping the randomized agent), at 6:30 hours (30 minutes after stopping therapy [crossover baseline]), and at 7:00 hours (30 minutes after starting the crossover agent) (Figure 1). After recording of the hemodynamic and oxygenation parameters, the originally randomized agent was re-started and continued or weaned per clinicians' decision. The collected hemodynamic parameters included systemic arterial pressure (systolic, diastolic, and mean), PA pressure (systolic, diastolic, and mean), CVP, cardiac index, $\mathrm{SvO}_{2}$, and heart rate (unless paced). Oxygenation parameters included $\mathrm{PO}_{2}$, $\mathrm{FIO}_{2}$, and oxygen saturation. All adverse events (methemoglobinemia, hypotension $[>30 \%$ decrease in mean blood pressure, unrelated to other measures], nonsurgical bleeding requiring reexploration, and complications of the delivery system [ie, sticking of the ventilator valve]) were prospectively recorded.

\section{Studied Agents}

NO was delivered from a tank at a concentration of 10,000 ppm diluted in nitrogen (Ikaria, Clinton, NJ). The desired concentration was regulated by a calibrated flow meter (Ikaria) and then delivered into the inspiratory limb of the ventilator. Peak inspired $\mathrm{NO}$ and $\mathrm{NO}_{2}$ concentrations were continuously analyzed by chemiluminescence (Ikaria). Exhaust gas was scavenged via a soda-lime absorber. Methemoglobin concentration (as a percentage of total hemoglobin) was measured at 6-hour intervals or more frequently if clinically indicated.

PGI2 (Flolan, Glaxo Wellcare Inc, Research Triangle Park, NC) was reconstituted in glycine buffer diluent to a final concentration of 20,000 ng/ $\mathrm{mL}$ by the pharmacy service and then delivered to the Respiratory Care Service. The nebulizer chamber was initially primed with $15 \mathrm{~mL}$ of PGI2 solution, and then a $60-\mathrm{mL}$ syringe of PGI2 was attached to an intravenous pump to be delivered at a rate of $8 \mathrm{~mL} / \mathrm{h}$ to the nebulizer chamber. The nebulizer system (Mini HEART nebulizer, Westmed, Tucson, AZ) was attached to the inspiratory limb of the ventilator circuit. At an oxygen flow rate of 
2 to $3 \mathrm{~L} / \mathrm{min}$, approximately $8 \mathrm{~mL}$ of PGI2 was nebulized per hour. A respiratory therapist familiar with the system was readily available during administration of PGI2.

\section{Statistical Analysis}

All data are presented as mean \pm standard error of the mean, unless otherwise indicated. Statistical analyses of the changes in hemodynamic and oxygenation parameters were performed using paired $t$ tests. By assuming a minimal detectable difference in mean PA pressure between the 2 treatment arms of at least $4 \mathrm{~mm} \mathrm{Hg}$, a patient standard deviation of $4 \mathrm{~mm} \mathrm{Hg}$, and a significance level of .05 , a minimum of 24 patients are necessary to detect a difference, with more than $90 \%$ power.

\section{RESULTS}

\section{Demographic and Clinical Data}

The demographic and clinical data are presented in Table 1. Thoracic transplant recipients $(\mathrm{n}=32)$ were randomized by initial treatment. Of the 32 randomized patients, 25 were included in the study (Figure 2). The 7 patients who were excluded failed to complete the study protocol; 6 patients (3 received NO and 3 received PGI2) were taken off the pulmonary vasodilator because of early clinical improvement, and 1 patient in the PGI2 group became hemodynamically unstable because of mediastinal bleeding after heart transplantation and the agent was discontinued. Reexploration revealed a surgical bleeding site. Therapy was switched to the other agent at crossover in all 25 study patients, so each patient received both $\mathrm{NO}$ and PGI2, and randomization determined only which agent was started first. The inhaled pulmonary vasodilator therapy was initiated for pulmonary hypertension in all patients $(\mathrm{n}=$ 25 ), with some patients also having refractory hypoxemia or RV dysfunction (Table 1).

\section{Nitric Oxide and Inhaled Prostacyclin Are Effective in Improving Hemodynamics (at Initiation of Therapy)}

The hemodynamic data for the study patients before any inhaled pulmonary vasodilator ( 0 minutes $)$ are presented for the cohort first treated with NO $(n=14)$ or PGI2 $(n=11)$ in Table 2. The initial responses to the pulmonary vasodilators after 30 minutes also are shown. NO and PGI2 treatments resulted in significant reductions in PA pressures and in CVP, increased cardiac index, and improved $\mathrm{SvO}_{2}$ after 30 minutes. Both agents showed no significant effects on $\mathrm{PaO}_{2} / \mathrm{FiO}_{2}$ ratio or systemic arterial blood pressure after 30 minutes.

\section{Nitric Oxide and Prostacyclin Similarly Reduce Postoperative Pulmonary Artery Pressures (at 6-Hour Crossover Trial)}

As noted in the "Materials and Methods" section, the hemodynamic and oxygenation parameters were measured after 6 hours of therapy with the initially randomized agent, after 30 minutes of washout (crossover baseline), and after 30 minutes of therapy with the crossover agent (Figure 1).
TABLE 1. Demographic and clinical data

\begin{tabular}{lc}
\hline Study group & 25 patients \\
Groups randomized by initial treatment & \\
NO & 14 patients \\
PGI2 & 11 patients \\
Age (y) & $59 \pm 2$ \\
Gender & \\
Male & 20 \\
Female & 5 \\
Procedure & \\
Single-lung transplant & 17 \\
$\quad$ Double-lung transplant & 2 \\
$\quad$ Orthotopic heart transplant & 6 \\
Indication for pulmonary vasodilator treatment ${ }^{\mathrm{a}}$ & \\
Pulmonary hypertension & 25 \\
Refractory hypoxemia & 5 \\
$\quad$ RV dysfunction & 6 \\
\hline NO, Nitric oxide; $P G I 2$, prostacyclin; $R V$, right ventricular. ${ }^{\mathrm{a}}$ More than 1 indication \\
was present for some patients.
\end{tabular}

During the postoperative crossover protocol, NO and PGI2 resulted in significant reductions in PA pressures compared with the crossover baseline (Figure 3,A). NO decreased the systolic PA pressure (NO: $30 \pm 1$ vs crossover baseline: $46 \pm 2 \mathrm{~mm} \mathrm{Hg}, P<.01$ ), diastolic PA pressure (NO: $17 \pm 1$ vs crossover baseline: $25 \pm 1 \mathrm{~mm} \mathrm{Hg}, P<$ .01 ), and PA mean pressure (NO: $23 \pm 1$ vs crossover baseline: $34 \pm 1 \mathrm{~mm} \mathrm{Hg}, P<.01)$. PGI2 treatment also reduced the systolic PA pressure (NO: $28 \pm 1$ vs crossover baseline: $46 \pm 2 \mathrm{~mm} \mathrm{Hg}, P<.01$ ), diastolic PA pressure (NO: $16 \pm 1$ vs crossover baseline: $25 \pm 1 \mathrm{~mm} \mathrm{Hg}, P<.01$ ), and mean PA pressure (NO: $22 \pm 1$ vs crossover baseline: $34 \pm 1$ $\mathrm{mm} \mathrm{Hg}, P<.01)$.

More important, when inhaled NO and PGI2 were compared, they had similar effects on PA pressure reduction at 6 hours. When the magnitude of the differences between the PA pressures and the crossover baseline $(\Delta$ PA pressure $=$ PA pressure - baseline) were compared, the $\Delta$ PA pressures for NO and PGI2 were similar (Figure 3, $B$ ). For NO and PGI2, the $\Delta$ systolic PA pressures were $16 \pm 2 \mathrm{~mm} \mathrm{Hg}$ (95\% confidence interval $[\mathrm{CI}], 11-20)$ and $17 \pm 2 \mathrm{~mm} \mathrm{Hg}$ (95\% CI, 13-22), respectively $(P=.10)$. The $\Delta$ diastolic PA pressures for NO and PGI2 were $8 \pm 1 \mathrm{~mm} \mathrm{Hg}(95 \%$ CI, 6-11) and $9 \pm 1 \mathrm{~mm} \mathrm{Hg}(95 \% \mathrm{CI}, 7-12)$, respectively $(P=.12)$. The $\Delta$ mean PA pressures for NO and PGI2 were $12 \pm 1 \mathrm{~mm} \mathrm{Hg}(95 \% \mathrm{CI}, 9-15)$ and $13 \pm 1 \mathrm{~mm} \mathrm{Hg}(95 \%$ CI, 9-16), respectively $(P=.32)$.

\section{Nitric Oxide and Prostacyclin Also Similarly \\ Decrease Central Venous Pressure, Increase Cardiac Index, and Improve Mixed Venous Oxygen Saturation (at 6-Hour Crossover Trial)}

During the postoperative crossover protocol, both $\mathrm{NO}$ and PGI2 significantly improved the hemodynamics compared 


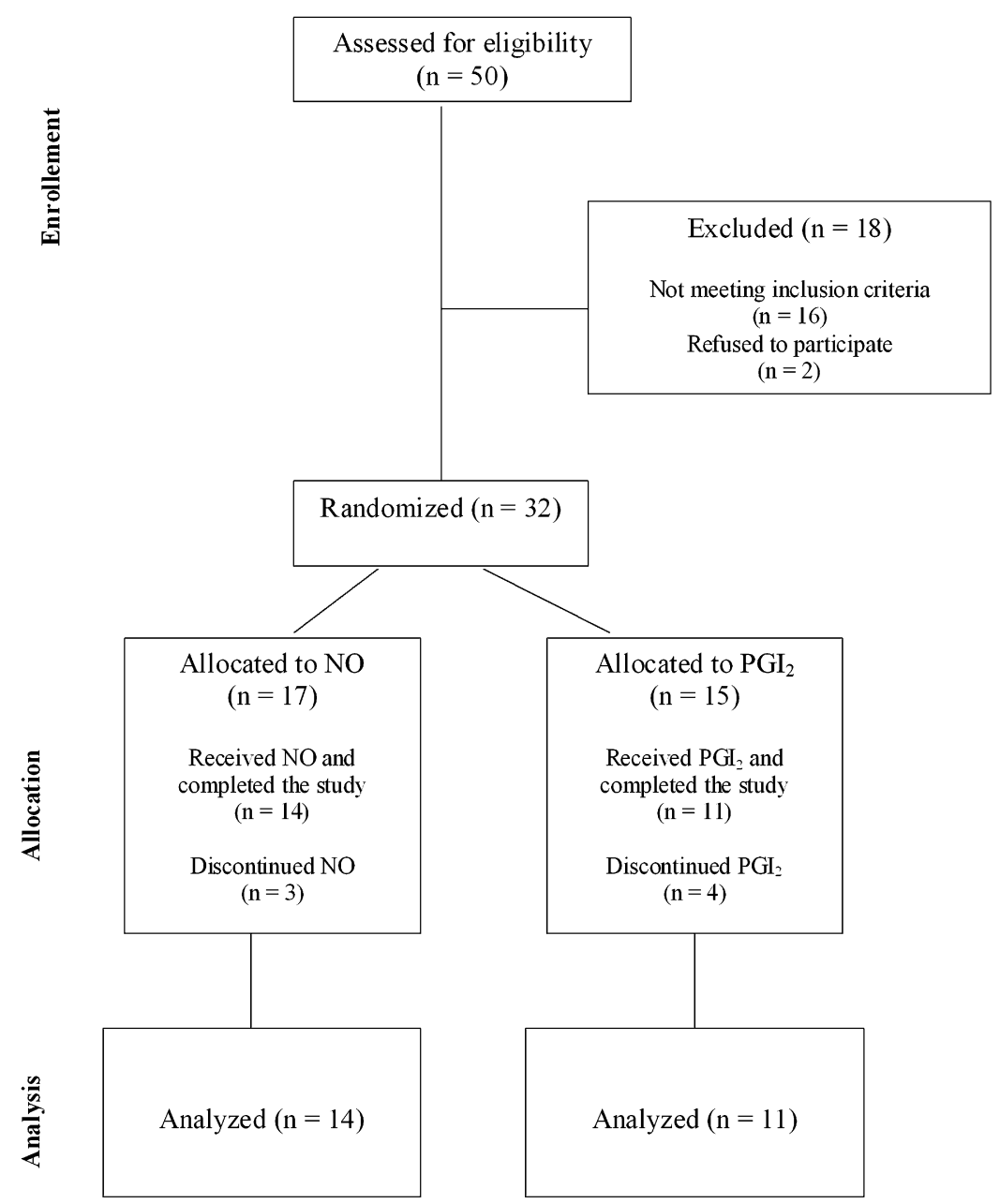

FIGURE 2. Flow diagram depicting the outcome of all screened and enrolled patients. NO, Nitric oxide; PGI2, prostacyclin.

with the crossover baseline (Figure 4). NO and PGI2 decreased the CVP (NO: $10 \pm 1$ vs crossover baseline: $14 \pm$ $1 \mathrm{~mm} \mathrm{Hg}, P<.01$; PGI2: $9 \pm 1$ vs crossover baseline: 14 $\pm 1 \mathrm{~mm} \mathrm{Hg}, P<.01)$. NO and PGI2 also increased the cardiac index (NO: $3.0 \pm 0.1$ vs crossover baseline: $2.5 \pm 0.1$ $\mathrm{L} / \mathrm{min} / \mathrm{m}^{2}, P<.01$; PGI2: $3.1 \pm 0.2$ vs crossover baseline: $\left.2.5 \pm 0.1 \mathrm{~L} / \mathrm{min} / \mathrm{m}^{2}, P<.01\right)$ and $\mathrm{SvO}_{2}(\mathrm{NO}: 72 \pm 2$ vs crossover baseline: $66 \pm 1 \%, P<.01$; PGI2: $71 \pm 2$ vs crossover baseline: $66 \% \pm 1 \%, P<.01)$.

Moreover, when the magnitude of the differences between the hemodynamic values and the crossover baseline values were determined (eg, $\Delta \mathrm{CVP}=\mathrm{CVP}$ - baseline), the $\Delta$ hemodynamic results were comparable. For NO and PGI2, the $\Delta \mathrm{CVP}$ values were $4 \pm 1 \mathrm{~mm} \mathrm{Hg}(95 \% \mathrm{CI}$, $3-5)$ and $5 \pm 1 \mathrm{~mm} \mathrm{Hg}(95 \% \mathrm{CI}, 3-6)$, respectively $(P=$ $.36)$. The $\Delta$ cardiac index values were $0.6 \pm .01 \mathrm{~L} / \mathrm{min} / \mathrm{m}^{2}$ (95\% CI, 0.4-0.9) and $0.8 \pm 0.1 \mathrm{~L} / \mathrm{min} / \mathrm{m}^{2}$ (95\% CI, 0.5$1.1)$, respectively $(P=.40)$. Finally, the $\Delta \mathrm{SvO}_{2}$ values were $7 \% \pm 1 \%(95 \% \mathrm{CI}, 4-9)$ and $7 \% \pm 1 \%(95 \% \mathrm{CI}$, 4-10), respectively $(P=.91)$.
Nitric Oxide and Prostacyclin Treatments Demonstrate No Significant Alterations in Postoperative Oxygenation or Systemic Arterial Blood Pressure (at 6-Hour Crossover Trial)

During the postoperative crossover protocol, NO showed no significant reduction in systemic arterial blood pressures (systolic: $104 \pm 3$ vs crossover baseline: $102 \pm 3 \mathrm{~mm} \mathrm{Hg}$, $P=.46$; diastolic: $58 \pm 2$ vs crossover baseline: $57 \pm 2 \mathrm{~mm}$ $\mathrm{Hg}, P=.30$; and mean: $73 \pm 2$ vs crossover baseline: $72 \pm$ $2 \mathrm{~mm} \mathrm{Hg}, P=.62)$. PGI2 also resulted in no significant decrease in systemic blood pressure (systolic: $99 \pm 3$ vs crossover baseline: $102 \pm 3 \mathrm{~mm} \mathrm{Hg}, P=.24$; diastolic: $55 \pm 2$ vs crossover baseline: $57 \pm 2 \mathrm{~mm} \mathrm{Hg}, P=.13$; and mean: $71 \pm 2$ vs crossover baseline: $72 \pm 3 \mathrm{~mm} \mathrm{Hg}, P=.63$ ). NO and PGI2 did not improve the $\mathrm{PaO}_{2} / \mathrm{FIO}_{2}$ ratio compared with the crossover baseline (NO: $269 \pm 25$ vs crossover baseline: $250 \pm 24, P=.06$; PGI2: $256 \pm 27$ vs crossover baseline: $250 \pm 24, P=.77$ ). In addition, there were no differences in $\mathrm{PaO}_{2} / \mathrm{FIO}_{2}$ ratio or systemic blood pressures when $\mathrm{NO}$ was compared with PGI2 (all $P=$ not significant, NO vs PGI2). 
TABLE 2. Initial response to nitric oxide and prostacyclin was similar

\begin{tabular}{|c|c|c|c|c|}
\hline & & $\begin{array}{l}\text { O } \\
=14)\end{array}$ & & $\begin{array}{l}\text { SI2 } \\
=11)\end{array}$ \\
\hline & 0 min & $30 \mathrm{~min}$ & 0 min & $30 \mathrm{~min}$ \\
\hline PA systolic (mm Hg) & $43 \pm 2$ & $36 \pm 2^{\mathrm{a}}$ & $51 \pm 2$ & $38 \pm 2^{\mathrm{a}}$ \\
\hline PA diastolic (mm Hg) & $24 \pm 1$ & $19 \pm 1^{\mathrm{a}}$ & $27 \pm 3$ & $21 \pm 2^{\mathrm{a}}$ \\
\hline PA mean $(\mathrm{mm} \mathrm{Hg})$ & $32 \pm 1$ & $26 \pm 1^{\mathrm{a}}$ & $37 \pm 3$ & $28 \pm 3^{\mathrm{a}}$ \\
\hline $\mathrm{CVP}(\mathrm{mm} \mathrm{Hg})$ & $14 \pm 1$ & $11 \pm 1^{\mathrm{a}}$ & $14 \pm 1$ & $10 \pm 1^{\mathrm{a}}$ \\
\hline $\begin{array}{l}\text { Cardiac } \\
\quad \text { index }\left(\mathrm{L} / \mathrm{min} / \mathrm{m}^{2}\right)\end{array}$ & $2.5 \pm 0.2$ & $3.0 \pm 0.3^{\mathrm{a}}$ & $2.6 \pm 0.2$ & $3.0 \pm 0.9^{\mathrm{a}}$ \\
\hline $\mathrm{SvO}_{2}(\%)$ & $69 \pm 3$ & $77 \pm 2^{\mathrm{a}}$ & $71 \pm 2$ & $76 \pm 3^{\mathrm{a}}$ \\
\hline $\mathrm{PaO}_{2} / \mathrm{FIO}_{2}$ ratio & $295 \pm 42$ & $330 \pm 35$ & $304 \pm 51$ & $360 \pm 59$ \\
\hline BP systolic (mm Hg) & $97 \pm 3$ & $88 \pm 3$ & $95 \pm 4$ & $95 \pm 3$ \\
\hline BP diastolic (mm Hg) & $59 \pm 2$ & $56 \pm 4$ & $57 \pm 3$ & $55 \pm 3$ \\
\hline $\mathrm{BP}$ mean $(\mathrm{mm} \mathrm{Hg})$ & $72 \pm 3$ & $66 \pm 3$ & $71 \pm 3$ & $69 \pm 3$ \\
\hline
\end{tabular}

\section{Clinical Outcomes}

The 30-day survival of this cohort of patients was $100 \%$. The median intensive care unit stay was 3 days. None of the 25 patients who completed this study required reexploration for bleeding. None of the heart transplant recipients required augmentation of therapy for pulmonary hypertension or RV dysfunction (ie, insertion of intra-aortic balloon pump, opening of the chest, or insertion of RV assist device). The incidence of PGD (grade 3) among lung transplant recipients at 48 hours was $5.3 \%$. There were no complications related to the PGI2 delivery system or PGI2 (systemic hypotension, flushing, nonsurgical bleeding), and we did not observe any toxicity related to $\mathrm{NO}$ administration (methemoglobinemia).

\section{DISCUSSION}

This study demonstrates that inhaled NO and PGI2 are equally effective in reducing the PA pressure and CVP, and increasing cardiac index and $\mathrm{SvO}_{2}$ at a crossover trial in a cohort of heart transplant and lung transplant recipients. Moreover, there were no complications of the PGI2 delivery system or toxicity associated to either agent during this study. An additional finding of this study is that in heart and lung transplant recipients with pulmonary hypertension, $\mathrm{NO}$ and inhaled PGI2 can reduce PA pressures and CVP, and improve cardiac index and $\mathrm{SvO}_{2}$ immediately after initiation of therapy. This latter observation is consistent with previous reports showing that inhaled NO improves pulmonary hemodynamics and oxygenation in thoracic transplant recipients. ${ }^{5-9}$

Inhaled PGI2 has also been demonstrated to be an effective treatment for pulmonary hypertension, RV dysfunction, and hypoxemia in cardiothoracic surgery. ${ }^{12,14}$ Haraldsson and colleagues ${ }^{12}$ reported the efficacy of inhaled PGI2 in producing a dose-dependent decrease in PVR, transpulmonary gradient, and CVP after cardiac surgery or heart trans-
PA Pressures

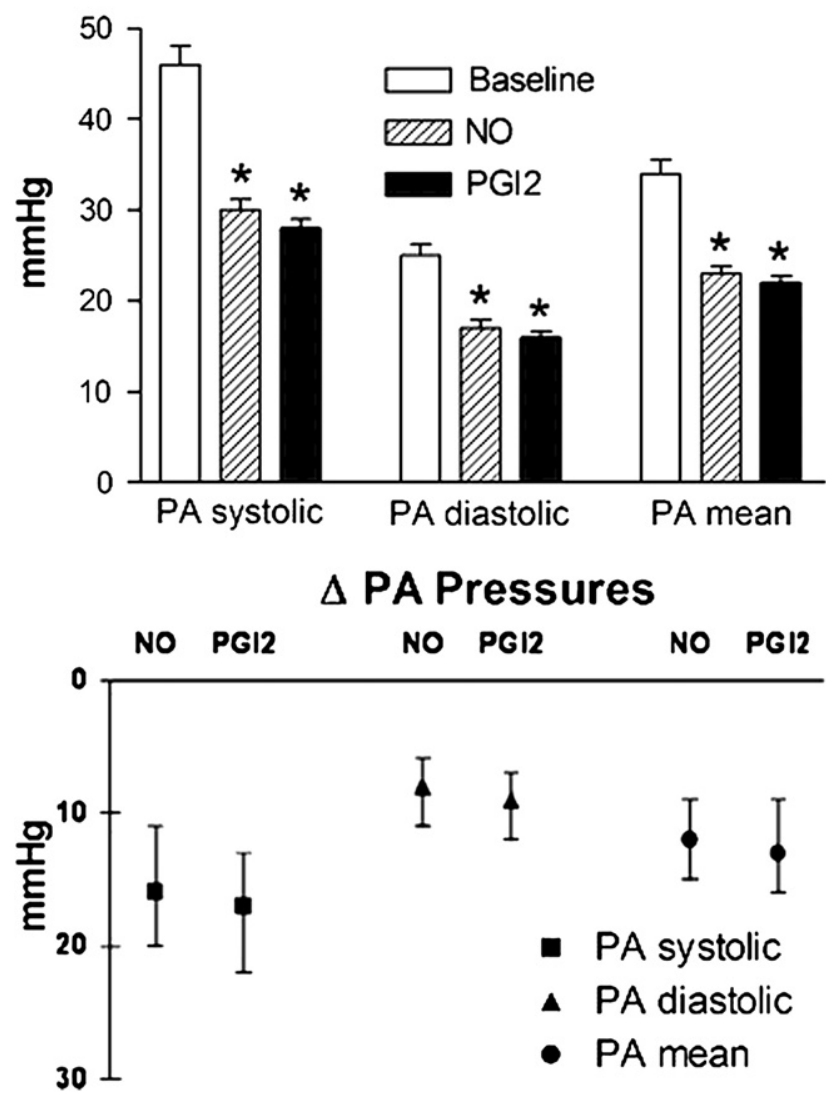

FIGURE 3. NO and PGI2 similarly reduce PA pressures at the 6-hour crossover trial. NO and PGI2 showed significant reductions in systolic, diastolic, and mean PA pressures compared with crossover baseline $\left({ }^{*} P<.01\right) . N O$, Nitric oxide; $P A$, pulmonary artery; $P G I 2$, prostacyclin.

plantation. In lung transplantation, inhaled PGI2 has been described as an effective alternative to NO in the treatment of pulmonary hypertension. ${ }^{14}$ Moreover, a prospective cohort study of 126 consecutive patients who underwent cardiothoracic surgery, including 12 heart and 43 lung transplant recipients, investigated the use of inhaled PGI2. Patients in this study with a mean PA pressure greater than $30 \mathrm{~mm} \mathrm{Hg}, \mathrm{PaO}_{2} / \mathrm{FIO}_{2}$ ratio less than 150 , or CVP greater than $16 \mathrm{~mm} \mathrm{Hg}$ with a cardiac index less than $2.2 \mathrm{~L} / \mathrm{min} /$ $\mathrm{m}^{2}$ were treated with inhaled PGI2. In this cohort analysis, inhaled PGI2 decreased PA pressures, increased oxygenation, and improved RV function. ${ }^{17}$

The central message from this report is that inhaled NO and PGI2 are equivalent in their hemodynamic effects at 6 hours after initiation of therapy in heart transplant and lung transplant recipients. Considering the findings of this comparison study and prior reports noted above, there is now growing evidence for the use of inhaled PGI2 as an alternative agent to $\mathrm{NO}$ in the treatment of pulmonary hypertension in thoracic transplantation. Inhaled PGI2 offers several advantages over NO that include a relative lack of 

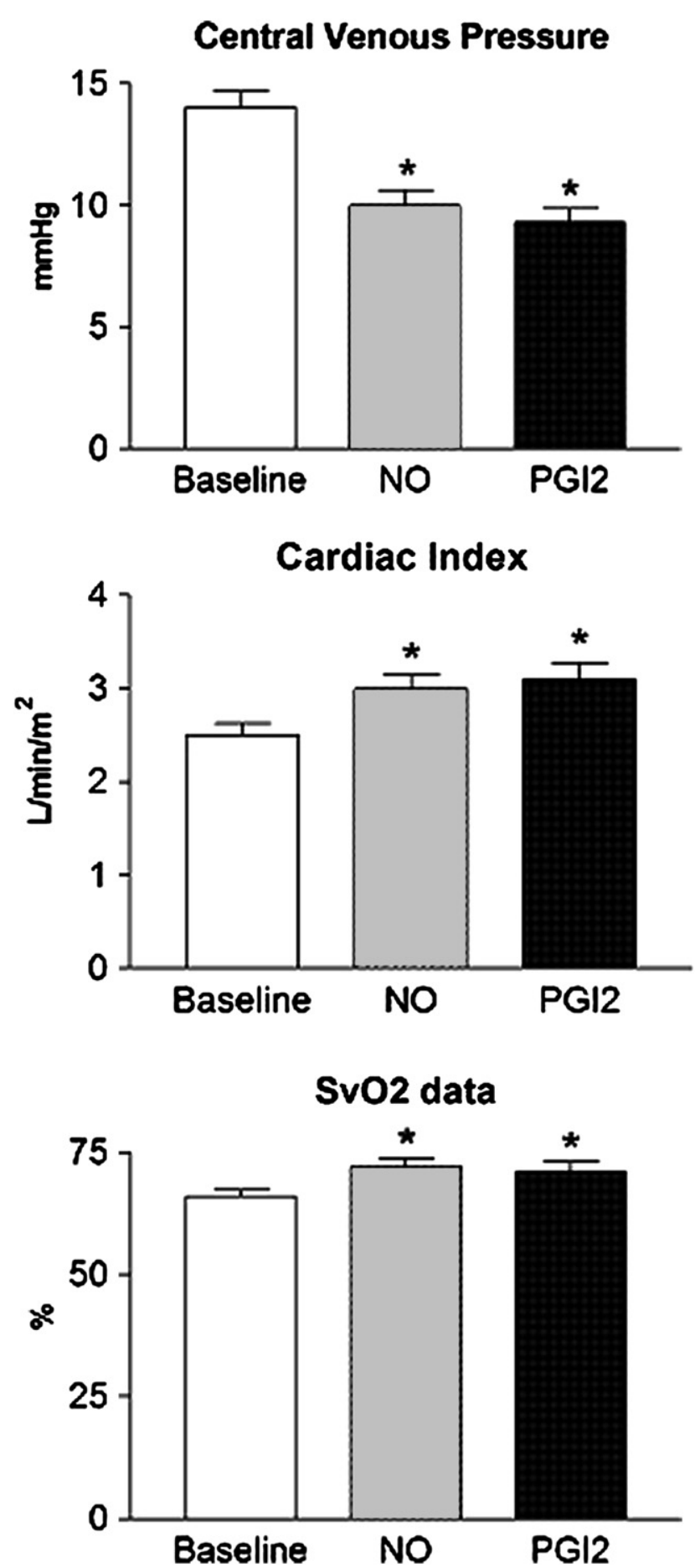

FIGURE 4. NO and PGI2 similarly improve hemodynamics at the 6-hour crossover trial. NO and PGI2 therapy decreased CVP and improved cardiac index and $\mathrm{SvO}_{2}$ when compared with crossover baseline values $(* P<.01)$. $\mathrm{NO}$, Nitric oxide; $P G I 2$, prostacyclin; $\mathrm{SvO}_{2}$, venous oxygen saturation.

toxicity and ease of delivery. NO requires specialized delivery systems and has known toxicities, such as methemoglobinemia, which limit the utility of the agent. ${ }^{10,18}$ However, toxicity caused by methemoglobinemia was not seen in this study. Another benefit is that PGI2 is less expensive than NO.

Although PGI2 offers a potentially less toxic, cheaper alternative to NO, there are disadvantages of PGI2. Systemic hypotension and flushing have been associated with inhaled PGI2, although these side effects were not observed in our study. Another concern is the risk of bleeding with inhaled PGI2 therapy, potentially related to platelet inhibition. ${ }^{19}$ One patient who was initially randomized to inhaled PGI2 was taken off the drug immediately and excluded from the study with the development of hypotension caused by surgical bleeding. There was no indication that the bleeding was due to inhaled PGI2 administration. In a prospective, double-blind, randomized study of 28 patients undergoing elective cardiac surgery, inhaled PGI2 resulted in impaired platelet function in vitro; however, there was no significant difference in chest tube output or hematocrit between control and treatment groups. ${ }^{20}$ Finally, there are technical issues involving the nebulizer delivery system and the mechanical ventilator. An inadvertent bolus of PGI2 as the result of tipping of the nebulizer is possible, which is a particular concern during transport from the operating room to the intensive care unit. Because of the sticky nature of the buffering agent, there is a danger of sticking of the ventilator valves, which is prevented by the required frequent changing of filters. During this study, no technical complications with the delivery of inhaled PGI2 or mechanical ventilator valves occurred. During inhaled PGI2 treatment, the availability of a respiratory therapist is required to monitor the nebulizer delivery system and ventilator, as well as frequently change the ventilator filters.

Although this study demonstrates that inhaled NO and PGI2 are equally effective in reducing PA pressures and improving other hemodynamic parameters, there was no significant improvement in oxygenation, as reported by several prior studies. ${ }^{7,8,21}$ One factor that may explain these findings is that only 5 of the 25 patients in this study had a $\mathrm{PaO}_{2} / \mathrm{FIO}_{2}$ ratio less than 150 . The mean $\mathrm{PaO}_{2} / \mathrm{FiO}_{2}$ ratio at the crossover baseline was 250 . In the 5 patients who had a $\mathrm{PaO}_{2} / \mathrm{FIO}_{2}$ ratio less than 150 , there was an approximately $50 \%$ increase in the $\mathrm{PaO}_{2} / \mathrm{FIO}_{2}$ ratio for both NO and PGI2 therapy. A larger number of patients with hypoxemia may have shown a statistically significant effect on oxygenation in this study.

A potential concern at the inception of this study was the rebound pulmonary hypertension at the crossover period. Although the PA pressures increased in nearly all patients at the time of crossover, none became hemodynamically unstable. In patients with difficulty weaning from NO, sildenafil has been shown to reduce PA pressures and allow weaning from the inhaled pulmonary vasodilator therapy. ${ }^{22,23}$ Sildenafil, a phosphodiesterase type 5 inhibitor, is an oral pulmonary vasodilator that prevents the breakdown of cGMP. ${ }^{24,25}$ Sildenafil was used liberally in this cohort of patients to facilitate weaning from NO or inhaled PGI2, after completion of the study. 


\section{LIMITATIONS}

There are several limitations of this study that deserve mention. a) There were many confounding variables that may have affected the oxygenation and hemodynamic parameters. These variables may have included the intraoperative and postoperative management of the ventilator, inotropic agents, vasodilators, and organ function. The management of these aspects of care was left under the control of the clinicians in the operating room and the intensive care unit. The optimization of the intraoperative and postoperative care directly by the clinicians was the safest way to perform the study without sacrificing patient care. b) Another limitation was the timing of the study. Our direct crossover comparison of $\mathrm{NO}$ and PGI2 was performed during the postoperative period, 6 hours after starting inhaled pulmonary vasodilator therapy. Because of the relative instability of thoracic transplant recipients in the operating room compared with 6 hours later, a crossover of agents at 30 minutes was not considered a safe experimental protocol. c) Another limitation of this study is the small number of studied subjects (especially heart transplant recipients). d) The findings of this study are only applicable to heart transplant and lung transplant recipients with "moderate" pulmonary hypertension, in the early postoperative periods. Caution needs to be exercised in the application of these findings to other patient populations (eg, adult patients undergoing cardiac surgery or pediatric patients). e) Finally, with the nebulizer method of drug delivery for inhaled PGI2, the exact amount of agent that reaches the alveoli is uncertain because of losses in the nebulizer chamber and ventilator tubing, and may vary in each patient (eg, depending on the mode of ventilation, settings, temperature). ${ }^{12}$

\section{CONCLUSIONS}

This prospective, randomized, crossover pilot study of inhaled pulmonary vasodilators in thoracic transplant recipients demonstrates that $\mathrm{NO}$ and inhaled PGI2 provide equivalent reductions in PA pressures and improvements in other hemodynamic parameters. Therefore, PGI2 may offer an alternative to NO in the treatment of pulmonary hypertension in thoracic transplantation.

\section{References}

1. Taylor DO, Edwards LB, Boucek MM, Trulock EP, Aurora P, Christie J, et al. Registry of the International Society for Heart and Lung Transplantation: twenty-fourth official adult heart transplant report-2007. J Heart Lung Transplant. 2007;26:769-81.

2. Griepp RB, Stinson EB, Dong E Jr, Clark DA, Shumway NE. Determinants of operative risk in human heart transplantation. Am J Surg. 1971;122:192-7.

3. Whitson BA, Prekker ME, Herrington CS, Whelan TP, Radosevich DM, Hertz MI, et al. Primary graft dysfunction and long-term pulmonary function after lung transplantation. J Heart Lung Transplant. 2007;26:1004-11.

4. Arcasoy SM, Fisher A, Hachem RR, Scavuzzo M, Ware LB. Report of the ISHLT Working Group on Primary Lung Graft Dysfunction part V: predictors and outcomes. J Heart Lung Transplant. 2005;24:1483-8.
5. Ardehali A, Hughes K, Sadeghi A, Esmailian F, Marelli D, Moriguchi J, et al. Inhaled nitric oxide for pulmonary hypertension after heart transplantation. Transplantation. 2001;72:638-41.

6. Kieler-Jensen N, Lundin S, Ricksten SE. Vasodilator therapy after heart transplantation: effects of inhaled nitric oxide and intravenous prostacyclin, prostaglandin E1, and sodium nitroprusside. J Heart Lung Transplant. 1995;14:436-43.

7. Ardehali A, Laks H, Levine M, Shpiner R, Ross D, Watson LD, et al. A prospective trial of inhaled nitric oxide in clinical lung transplantation. Transplantation. 2001;72:112-5.

8. Date H, Triantafillou AN, Trulock EP, Pohl MS, Cooper JD, Patterson GA. Inhaled nitric oxide reduces human lung allograft dysfunction. J Thorac Cardiovasc Surg. 1996;111:913-9.

9. Adatia I, Lillehei C, Arnold JH, Thompson JE, Palazzo R, Fackler JC, et al. Inhaled nitric oxide in the treatment of postoperative graft dysfunction after lung transplantation. Ann Thorac Surg. 1994;57:1311-8.

10. Kirmse M, Hess D, Fujino Y, Kacmarek RM, Hurford WE. Delivery of inhaled nitric oxide using the Ohmeda INOvent Delivery System. Chest. 1998;113: 1650-7.

11. Welte M, Zwissler B, Habazettl H, Messmer K. PGI2 aerosol versus nitric oxide for selective pulmonary vasodilation in hypoxic pulmonary vasoconstriction. Eur Surg Res. 1993;25:329-40.

12. Haraldsson A, Kieler-Jensen N, Ricksten SE. Inhaled prostacyclin for treatment of pulmonary hypertension after cardiac surgery or heart transplantation: a pharmacodynamic study. J Cardiothorac Vasc Anesth. 1996;10:864-8.

13. Haraldsson A, Kieler-Jensen N, Nathorst-Westfelt U, Bergh CH, Ricksten SE Comparison of inhaled nitric oxide and inhaled aerosolized prostacyclin in the evaluation of heart transplant candidates with elevated pulmonary vascular resistance. Chest. 1998;114:780-6.

14. Fiser SM, Cope JT, Kron IL, Kaza AK, Long SM, Kern JA, et al. Aerosolized prostacyclin (epoprostenol) as an alternative to inhaled nitric oxide for patients with reperfusion injury after lung transplantation. J Thorac Cardiovasc Surg. 2001;121:981-2

15. Mikhail G, Gibbs J, Richardson M, Wright G, Khaghani A, Banner N, et al. An evaluation of nebulized prostacyclin in patients with primary and secondary pulmonary hypertension. Eur Heart J. 1997;18:1499-504.

16. Walmrath D, Schneider T, Schermuly R, Olschewski H, Grimminger F, Seeger W. Direct comparison of inhaled nitric oxide and aerosolized prostacyclin in acute respiratory distress syndrome. Am J Respir Crit Care Med. 1996;153: 991-6.

17. De Wet CJ, Affleck DG, Jacobsohn E, Avidan MS, Tymkew H, Hill LL, et al. Inhaled prostacyclin is safe, effective, and affordable in patients with pulmonary hypertension, right heart dysfunction, and refractory hypoxemia after cardiothoracic surgery. J Thorac Cardiovasc Surg. 2004;127:1058-67.

18. Young JD, Dyar O, Xiong L, Howell S. Methaemoglobin production in normal adults inhaling low concentrations of nitric oxide. Intensive Care Med. 1994; 20:581-4.

19. Moncada S, Gryglewski R, Bunting S, Vane JR. An enzyme isolated from arteries transforms prostaglandin endoperoxides to an unstable substance that inhibits platelet aggregation. Nature. 1976;263:663-5.

20. Haraldsson A, Kieler-Jensen N, Wadenvik H, Ricksten SE. Inhaled prostacyclin and platelet function after cardiac surgery and cardiopulmonary bypass. Intensive Care Med. 2000;26:188-94

21. Meade MO, Granton JT, Matte-Martyn A, McRae K, Weaver B, Cripps P, et al A randomized trial of inhaled nitric oxide to prevent ischemia-reperfusion injury after lung transplantation. Am J Respir Crit Care Med. 2003;167:1483-9.

22. Trachte AL, Lobato EB, Urdaneta F, Hess PJ, Klodell CT, Martin TD, et al. Oral sildenafil reduces pulmonary hypertension after cardiac surgery. Ann Thorac Surg. 2005;79:194-7.

23. Namachivayam P, Theilen U, Butt WW, Cooper SM, Penny DJ Shekerdemian LS. Sildenafil prevents rebound pulmonary hypertension after withdrawal of nitric oxide in children. Am J Respir Crit Care Med. 2006;174: 1042-7.

24. Galie N, Ghofrani HA, Torbicki A, Barst RJ, Rubin LJ, Badesch D, et al. Sildenafil citrate therapy for pulmonary arterial hypertension. N Engl J Med. 2005;353: 2148-57.

25. Michelakis E, Tymchak W, Lien D, Webster L, Hashimoto K, Archer S. Oral sildenafil is an effective and specific pulmonary vasodilator in patients with pulmonary arterial hypertension: comparison with inhaled nitric oxide. Circulation. 2002;105:2398-403. 


\section{Discussion}

Dr Michael Mulligan (Seattle, Wash). I would like to thank the association for inviting me to discuss this paper and Dr Khan and his colleagues for sending me the manuscript in advance. Congratulations on a fine presentation and for conducting a prospective randomized trial in an attempt to answer a timely question. You added $50 \%$ more patients between submission of the abstract and the manuscript, so I paid attention and I think I understand your take-home message but let's work through this.

I have 2 comments and then 3 questions.

The first comment is that you combined heart and lung transplant patients and I do not think that is appropriate. That is apples and oranges, and I think you think so, too, because you use different entrance criteria to include heart or lung transplant recipients. Whereas pulmonary artery pressures and elevations in pulmonary vascular resistance have obvious implications for outcomes in heart transplant patients with right ventricular dysfunction, elevated pulmonary artery pressures are not even used in the grading criteria for primary graft dysfunction after lung transplantation. Rather, we use $\mathrm{P}$ to $\mathrm{F}$ ratios and infiltrates.

Second comment-you raised 3 concerns about inhaled nitric oxide: the need for complex delivery systems, potential toxicities, and cost. Yet in your data and in your discussion, you realize that both inhaled nitric oxide and nebulized prostacyclin require somewhat complex delivery systems and you observed no toxicity so it really comes down to cost. If we have already spent so much, on a lung transplantation for example, I think it is best to use the best drug with the best bioavailability in the alveolar space with the best effect on ventilation perfusion matching, accepting an increase in cost that is real but only a small fraction of the overall price of the successful patient outcome. Ultimately, we still do not appear to know which agent is best.

I will move on to the questions.

First question, there were 17 patients in the abstract and 25 patients in the manuscript. The numbers are really too small to draw any definitive conclusions about efficacy in heart transplant recipients or in lung transplant recipients. Was this your target or did you stop enrollment early for some reason?

Dr Khan. We initially had a target of 100 patients. However, when we did our preliminary analysis and reviewed this with we found reasonable data that we concluded the study early.

Dr Mulligan. I would challenge the fact that you hit a home run because hypoxia is - this is my second question. Hypoxia is the un- doing of a lung transplant recipient in primary graft dysfunction; yet, in your manuscript you showed no improvement in $\mathrm{P}$ to $\mathrm{F}$ ratios with either nebulized prostacyclin or inhaled nitric oxide. Many other studies have shown beneficial effects with both. This is especially true with inhaled nitric oxide, presumably related to the better alveolar distribution of a gas as compared with a nebulized solution. Why do you suppose that in your study you did not see any improvement in $\mathrm{P}$ to $\mathrm{F}$ ratios? Did it have something to do with the 8 additional patients added, because in your abstract you did see a benefit, or was it because you simply did not have enough acute graft dysfunction in lung transplant population?

Dr Khan. The difference between the data in the abstract and the manuscript with the larger numbers, the small difference in improvement in PF ratio became no longer significant. That was because in the group of patients that had a PF ratio of less than 200, when you looked at that subgroup, they had approximately $50 \%$ increase in PF ratio because their PF ratio started in the 50-100 range. However, when you look at the mean of the whole group, the average is 200-250. When we did look at that group separately, they did have an improvement in oxygenation from both agents. However, when we added more patients, none of whom had hypoxia, it diluted out that finding.

Dr Mulligan. I think that gets back to the original comment that we probably should not mix heart and lung transplant patients together in such a study and we should expand it to include more lung transplant patients.

The last question is that in your manuscript and in your presentation you talked about a 5.3\% incidence of grade III primary graft dysfunction in your lung recipients, yet 5 of 17 or 5 of 25 had $P$ to F ratios of less than 200 , which would imply a grade III primary graft dysfunction if they were lung transplant recipients. What was your actual incidence of primary graft dysfunction in this population?

Dr Khan. It was $5.3 \%$ at 48 hours. However, that initial data of the 5 patients who had hypoxemia initially was at the time of randomization in the operating room, so several of these patients improved during the next 48 hours such that there was only 1 patient remaining who met the criteria.

Dr Mulligan. There is an upfront and then there is a delayed assessment, so I do not know which one is valid but it appeared to be disparate between your early and late assessments.

Again congratulations and I appreciate the opportunity to discuss the paper.

Dr Khan. Thank you. 